\title{
Comparative effectiveness of flexible versus rigid neuroendoscopy for endoscopic third ventriculostomy and choroid plexus cauterization: a propensity score-matched cohort and survival analysis
}

\author{
Shelly Wang, MD, MPH, ${ }^{1,2}$ Scellig Stone, MD, PhD, FRCSC, ${ }^{3}$ Alexander G. Weil, MD, FRCSC, ${ }^{4}$ \\ Aria Fallah, MD, MSc, FRCSC, ${ }^{5,6}$ Benjamin C. Warf, MD, ${ }^{3}$ John Ragheb, MD, ${ }^{7}$ Sanjiv Bhatia, MD, ${ }^{7}$ \\ and Abhaya V. Kulkarni, MD, PhD, FRCSC ${ }^{1,8}$ \\ 'Division of Neurosurgery, Department of Surgery, University of Toronto, Ontario, Canada; ${ }^{2}$ Departments of Biostatistics and \\ Epidemiology, Harvard T. H. Chan School of Public Health, Boston; ${ }^{3}$ Department of Neurosurgery, Boston Children's Hospital \\ and Harvard Medical School, Boston, Massachusetts; ${ }^{4}$ Department of Neurosurgery, Sainte Justine Hospital, University of \\ Montreal, Quebec, Canada; ${ }^{5}$ Division of Pediatric Neurosurgery, Department of Surgery, David Geffen School of Medicine at \\ University of California, Los Angeles; ${ }^{6}$ Brain Research Institute, University of California, Los Angeles, California; ' ${ }^{7}$ Department \\ of Neurosurgery, Nicklaus Children's Hospital-Jackson Memorial Hospital, Miami, Florida; and ${ }^{8}$ Division of Neurosurgery, The \\ Hospital for Sick Children, University of Toronto, Ontario, Canada
}

\begin{abstract}
OBJECTIVE Endoscopic third ventriculostomy (ETV)/choroid plexus cauterization (CPC) has become an increasingly common technique for the treatment of infant hydrocephalus. Both flexible and rigid neuroendoscopy can be used, with little empirical evidence directly comparing the two. Therefore, the authors used a propensity score-matched cohort and survival analysis to assess the comparative efficacy of flexible and rigid neuroendoscopy.

METHODS Individual data were collected through retrospective review of infants younger than 2 years of age, treated at 1 of 2 hospitals: 1) Boston Children's Hospital, exclusively utilizing flexible neuroendoscopy, and 2) Nicklaus Children's Hospital-Jackson Memorial Hospital, exclusively utilizing rigid neuroendoscopy. Patient characteristics and postoperative outcomes were assessed. A propensity score model was developed to balance patient characteristics in the case mix.

RESULTS A propensity score model for neuroendoscope type was developed with 5 independent variables: chronological age, sex, hydrocephalus etiology, prior CSF diversion, and prepontine scarring. Propensity score decile-adjusted and 1-to-1 nearest-neighbor matching analysis revealed that compared with flexible neuroendoscopy, rigid neuroendoscopy had an ETV/CPC failure odds ratio (OR) of $1.43(p=0.31)$ and $1.31(p=0.47)$, respectively, compared with an unadjusted OR of $2.40(p=0.034)$. Furthermore, in a Cox regression analysis controlled by propensity score, rigid neuroendoscopy had a hazard ratio (HR) of 1.10 ( $p=0.70)$, compared with an unadjusted HR of $1.61(p=0.031)$.

CONCLUSIONS Although unadjusted analysis suggested worse ETVICPC outcomes for infants treated by rigid neuroendoscopy, much of the difference could be attributed to the case mix and other predictors of outcome. A larger sample observational study or randomized controlled trials are required to provide evidence-based guidelines on ETVICPC technique.
\end{abstract}

https://thejns.org/doi/abs/10.3171/2016.12.PEDS16443

KEY WORDS choroid plexus; endoscopic third ventriculostomy; hydrocephalus; neuroendoscope

I NFANT hydrocephalus is a major neurosurgical concern worldwide, and leads to significant morbidity and mortality. Cerebrospinal fluid shunts (i.e., ventriculoperitoneal shunts) remain the mainstay treatment for infant hydrocephalus; however, shunt-related complications, including shunt failure and infection, result in considerable morbidity. Endoscopic third ventriculostomy (ETV) can obviate the need for CSF shunts in many hydrocephalic infants, and the addition of choroid plexus cauterization (CPC) may increase its likelihood of success. Warf's ex-

ABBREVIATIONS $\mathrm{Cl}$ = confidence interval; $\mathrm{CPC}=$ choroid plexus cauterization; $\mathrm{ETV}=$ endoscopic third ventriculostomy; ETVSS = ETV Success Score; HR = hazard ratio; $\mathrm{OR}=$ odds ratio. 
perience over the past decade treating children in East Africa demonstrated the efficacy of ETV/CPC compared with ETV alone, especially among infants younger than 12 months of age., $, 12,14,15,18$ Over the past decade, ETV/ $\mathrm{CPC}$ has become an increasingly common treatment for infant hydrocephalus in Africa, North America, Asia, and Europe. ${ }^{2,4,5,8,20}$ Proposed mechanisms by which CPC promotes ETV success include decreasing CSF production and altering CSF dynamics. ${ }^{7,9-11}$

Both flexible and rigid neuroendoscopes can be used for CPC, and practice patterns vary between surgeons and centers. Currently, the choice between flexible and rigid neuroendoscopy is commonly dictated by surgeon familiarity and local availability of the instrument, and lacks guidance from evidence-based patient-related outcomes. The use of a flexible neuroendoscope, as originally described by Warf, allows for more extensive cauterization of the choroid plexus, particularly in bilateral anterior temporal horns of the lateral ventricle and theoretically less traction injury in the superficial cerebral cortex at the site of scope insertion. Its major limitation, however, is that many surgeons are less familiar with its use, resulting in a clinician operative learning curve. Rigid neuroendoscopes are more familiar to most neurosurgeons, but do not allow for extensive choroid plexus cauterization in the anterior temporal horns. Both types of neuroendoscopes are used across centers in North America for ETV/CPC, with reported 12-month success rates ranging from $38 \%$ for rigid neuroendoscopy ${ }^{20}$ to $57 \%$ for flexible neuroendoscopy. ${ }^{8}$ In Africa, Asia, and Europe, both flexible neuroendoscopy $y^{1,5,9,10,14,15,19}$ and rigid neuroendoscopy ${ }^{2}$ have been used, with no consensus on the superiority of either technique. A retrospective review of ETV/CPC treatment outcomes from multiple North American centers participating in the Hydrocephalus Clinical Research Network suggested that greater extent of CPC and the use of a flexible neuroendoscope were associated with improved success, although the sample size was small and the patients were not randomized to neuroendoscopy type. ${ }^{4}$ Furthermore, the extent of CPC was found to be an independent determinant of ETV/CPC success in 1 large study of Ugandan infants. ${ }^{17} \mathrm{~A}$ more recent study has correlated ETV/CPC success with the extent of CPC as evaluated by postoperative MRI. ${ }^{6}$

In this analysis, we aimed to assess the efficacy of flexible versus rigid neuroendoscopy for ETV/CPC for infants younger than 2 years of age with hydrocephalus treated in 2 high-volume pediatric neurosurgical centers in North America. Neuroendoscope type is center-specific and thus center-related factors (such as differences in patient selection) may confound outcomes. We therefore developed a propensity score model to balance the risk factors for the case mix.

\section{Methods \\ Study Population}

Individual participant data were collected from 2 recent retrospective published series from 2 separate institutions: 1) Boston Children's Hospital, exclusively utilizing flexible neuroendoscopy, ${ }^{8}$ and 2) Nicklaus Children's
Hospital-Jackson Memorial Hospital, exclusively utilizing rigid neuroendoscopy. ${ }^{20}$ Cohorts from these 2 centers were chosen, as they consist of the largest groups of patients for each neuroendoscopy type in North America. The inclusion criteria for the infants were the following: 1) younger than 2 years of chronological age at the time of surgery; 2) presented with hydrocephalus of any underlying etiology; 3) had undergone an ETV/CPC procedure; and 4) had at least 1 follow-up data point available (length of follow-up or time to repeat CSF diversion procedure).

\section{Data Collection}

Patient demographics, chronological age, hydrocephalus etiology, history of prior CSF diversion (previous reservoir, external ventricular drain, ventriculosubgaleal shunt, or ventriculoperitoneal shunt), presence of prepontine scarring (as determined intraoperatively by the surgeon), and postoperative outcome were collected and assessed. ETV/CPC failure was defined as the need for a second definitive hydrocephalus surgery with either repeat endoscopic treatment or shunt placement. A crude analysis of the ETV/CPC treatment failure of these 2 cohorts was performed using logistic regression and Cox proportional hazards regression survival analysis, to account for variable follow-up durations. A commonly used survival analysis method, the Cox model assumes that the relative failure rate (i.e., hazard ratio [HR]) of ETV/CPC remains constant over time between the 2 comparison cohorts.

\section{Propensity Score Model}

To help account for potential confounders, a propensity score model was developed, utilizing independent predictors of outcome and the dependent variable of neuroendoscopy type. Propensity scores predict the probability that a given patient, with a given set of covariates, would receive flexible neuroendoscopy rather than rigid neuroendoscopy. As such, it collapses the effect of the measured covariates into a single numerical value between 0 and 1 . Propensity score-adjusted analysis was completed using multiple techniques. The primary analysis was a propensity score-matched logistic regression analysis. Propensity score decile-adjusted analysis was performed, which was statistically robust and allowed inclusion of all participants. Additionally, 1-to-1 nearest-neighbor matching by propensity scores was also completed, which involved closely matched pairs of individuals from each neuroendoscopy type, and excluded outliers in each cohort. By matching subjects in the flexible and rigid neuroendoscopy cohorts by propensity score, any difference in outcome was assumed to be related to the ETV/CPC technique.

Secondary analyses included Cox proportional hazards regression controlled by propensity score and stratified by propensity score deciles. All statistics were completed using Stata/MP (version 13, StataCorp LP).

\section{Results}

\section{Patient Characteristics}

Data on a total of 91 infants from Boston Children's Hospital (surgery between August 2009 and March 2014) and 85 infants from Nicklaus Children's Hospital-Jackson 
Memorial Hospital (surgery between July 2007 and May 2014) were collected. The descriptive characteristics and the $\mathrm{p}$ value between the 2 cohorts, calculated by the Wilcoxon rank-sum test for nonparametric data, are presented in Table 1. There were no repeated ETV/CPC procedures in this cohort and only 1 data point per infant. All patients from Boston Children's Hospital underwent ETV/ CPC by flexible neuroendoscopy (Storz 3.7-mm flexible neuroendoscope) by a single surgeon (B.C.W.). The flexible neuroendoscopy technique involves a right frontal burhole approach, performance of a standard ETV, inspection and fenestration of any residual membranes including the Liliequist membrane, and bilateral CPC from the foramen of Monro to the anterior tip of the temporal horns using monopolar cautery, with fenestration of the septum pellucidum if necessary. ${ }^{8}$ All patients from Nicklaus Children's Hospital-Jackson Memorial Hospital underwent ETV/ CPC by rigid neuroendoscopy (Aesculap or Claris rigid neuroendoscope) by 1 of 4 pediatric neurosurgeons. The rigid neuroendoscopy technique involves a right frontal approach with similar steps to the flexible neuroendoscopy technique; however, the extent of CPC varied depending on ventricle size and septal anatomy. ${ }^{20}$

As a part of the neuroendoscopy technique, the extent of temporal horn CPC differed between the 2 cohorts. All of the infants in the flexible neuroendoscopy cohort underwent bilateral CPC. In contrast, 73 infants $(85.9 \%)$ in the rigid neuroendoscopy cohort underwent bilateral CPC and 12 infants (14.1\%) underwent unilateral CPC. Furthermore, in the rigid neuroendoscopy cohort, 17 infants (20.0\%) underwent no temporal horn CPC, 28 infants $(32.9 \%)$ underwent unilateral or incomplete temporal horn CPC, and 40 infants (47.1\%) underwent complete bilateral temporal horn CPC.

The pooled cohort (176 infants) consisted of $54 \%$ males and $46 \%$ females. The median (mean) chronological age

TABLE 1. Characteristics of infants undergoing ETVICPC by flexible and rigid neuroendoscopy

\begin{tabular}{|c|c|c|c|c|}
\hline \multirow[b]{2}{*}{ Characteristic } & \multicolumn{2}{|c|}{ Neuroendoscope } & \multirow[b]{2}{*}{ Total } & \multirow[b]{2}{*}{$p$ Value $^{*}$} \\
\hline & Flexible & Rigid & & \\
\hline No. of patients & 91 & 85 & 176 & \\
\hline Time interval & August 2009-March 2014 & July 2007-May 2014 & July 2007-May 2014 & \\
\hline Males (\%) & $50(54.9)$ & $45(52.9)$ & $95(54.0)$ & 0.40 \\
\hline \multicolumn{5}{|l|}{ Age at surgery (mos) } \\
\hline$<1(\%)$ & $25(27.5)$ & $19(22.4)$ & $44(25.0)$ & \\
\hline $1-6(\%)$ & $36(39.6)$ & $47(55.3)$ & $83(47.2)$ & \\
\hline $6-12(\%)$ & $23(25.3)$ & $18(21.2)$ & $41(23.3)$ & \\
\hline $12-24(\%)$ & $7(7.7)$ & $1(1.2)$ & $8(4.5)$ & \\
\hline Median (IQR) & $3.2(0.7-6.8)$ & $3.5(1.2-5.7)$ & $3.2(1.0-6.3)$ & 0.38 \\
\hline Complete CPC (\%) & $90(98.9)$ & $73(86.0)$ & $163(92.6)$ & 0.001 \\
\hline \multicolumn{5}{|l|}{ Temporal horn CPC (\%) } \\
\hline None & 0 & $17(20.0)$ & $17(9.7)$ & \multirow{3}{*}{$<0.001$} \\
\hline Unilat or incomplete & $1(1.1)$ & $28(32.9)$ & $29(16.5)$ & \\
\hline Bilat complete & $90(98.9)$ & $40(47.1)$ & $130(73.9)$ & \\
\hline \multicolumn{5}{|l|}{ Hydrocephalus etiology (\%) } \\
\hline Post-hemorrhagic hydrocephalus & $25(27.5)$ & $44(51.8)$ & $69(39.2)$ & \multirow{7}{*}{0.022} \\
\hline Aqueductal stenosis & $21(23.1)$ & $12(14.1)$ & $33(18.8)$ & \\
\hline Myelomeningocele & $23(25.3)$ & $7(8.2)$ & $30(17.0)$ & \\
\hline Dandy-Walker complex & $6(6.6)$ & $6(7.1)$ & $12(6.8)$ & \\
\hline Congenital idiopathic hydrocephalus & $4(4.4)$ & $6(7.1)$ & $10(5.7)$ & \\
\hline Postinfectious hydrocephalus & $6(6.6)$ & $5(5.9)$ & $11(6.3)$ & \\
\hline Other etiology & $6(6.6)$ & $5(5.9)$ & $11(6.3)$ & \\
\hline Prior CSF diversion (\%) & $17(18.9)$ & $33(38.8)$ & $50(28.4)$ & 0.032 \\
\hline Prepontine scarring (\%) & $15(16.5)$ & $25(29.4)$ & $40(22.7)$ & 0.041 \\
\hline Median ETVSS (IQR) & $40(30-60)$ & $40(40-50)$ & $40(40-50)$ & 0.87 \\
\hline \multicolumn{5}{|l|}{ Outcome } \\
\hline \% 12-mo success by Kaplan-Meier estimates & $57 \%$ & $38 \%$ & $48 \%$ & 0.0044 \\
\hline Median follow-up period in mos (IQR) $\dagger$ & $4.4(1.6-20.2)$ & $4.0(1.0-12.0)$ & $4.1(1.3-20.0)$ & 0.66 \\
\hline Operative morbidity (\%) & $2(2.2)$ & $5(5.9)$ & $7(4.0)$ & 0.21 \\
\hline
\end{tabular}

$\mathrm{IQR}=$ interquartile range.

Boldface type indicates statistical significance.

* $p$ values calculated by the Wilcoxon rank-sum test for nonparametric distributions.

$\dagger$ Simple median follow-up for all patients to minimum time of repeat surgery or censoring. 
at the time of surgery was 3.2 (4.3) months. Hydrocephalus etiology included posthemorrhagic hydrocephalus (39.2\%), myelomeningocele (17.0\%), Dandy-Walker complex (6.8\%), congenital idiopathic hydrocephalus (6.3\%), postinfectious hydrocephalus (6.3\%), congenital aqueductal stenosis (5.7\%), and other etiologies, including tectal tumors and cysts $(6.3 \%)$. In the pooled cohort, $28.4 \%$ of the infants had prior CSF diversion and $22.7 \%$ had prepontine scarring. The median Endoscopic Third Ventriculostomy Success Score (ETVSS) ${ }^{3}$ was 40. Pooled 12-month ETV/ CPC success by Kaplan-Meier estimate was $48 \%$ (95\% confidence interval [CI] 40\%-56\%).

For the cohort treated by flexible neuroendoscopy, there were 2 cases of operative morbidity (2.2\%), which included 1 CSF leak and 1 transient syndrome of inappropriate antidiuretic hormone secretion. For the cohort treated by rigid neuroendoscopy, there were 6 complications in 5 patients $(5.8 \%$ ), including 2 hygromas, $1 \mathrm{CSF}$ leak, and 3 infections. There were no perioperative deaths (within 30 days of surgery) in either cohort.

\section{Survival Analysis}

Nelson-Aalen cumulative hazard estimates of the entire cohort $(n=176)$ demonstrated highest rates of ETV/ CPC treatment failure in the 1st year after surgery (Fig. 1). Kaplan-Meier survival estimates demonstrated significant differences in treatment success for infants who underwent flexible versus rigid neuroendoscopy (Fig. 2). On crude analysis, rigid neuroendoscopy was associated with worse outcome, with an HR of 1.61 (95\% CI 1.05-2.48, p $=0.031)$ on Cox proportional hazards regression.

Univariate Cox regression analysis identified covariates to be predictors of survival. Etiology was a significant predictor of survival, with postinfectious hydrocephalus associated with the worst outcome (HR 3.48, 95\% CI 1.73-7.01, $\mathrm{p}=0.0027)$, and myelomeningocele associated with favorable outcomes (HR 0.49, 95\% CI 0.25-0.94, p $=0.019$; Fig. 3). Prior CSF diversion (previous reservoir, external ventricular drain, ventriculosubgaleal shunt, or ventriculoperitoneal shunt) was also a significant predictor of treatment failure (HR 1.78, 95\% CI 1.15-2.77, $\mathrm{p}=0.013$; Fig. 4), as was the presence of prepontine scarring (HR $2.80,95 \%$ CI 1.77-4.43, p < 0.0001; Fig. 5). Age older than

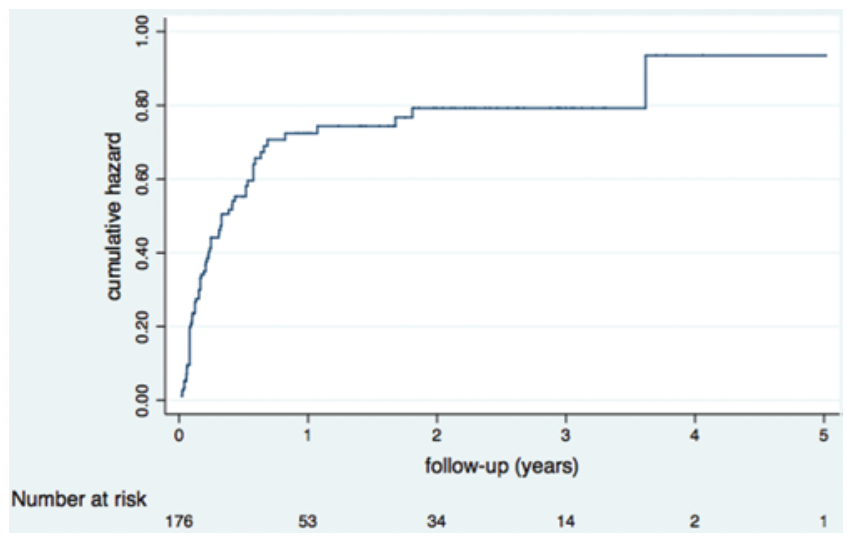

FIG. 1. Overall Nelson-Aalen estimates of cumulative hazard of failures for ETV/CPC failure. Figure is available in color online only.

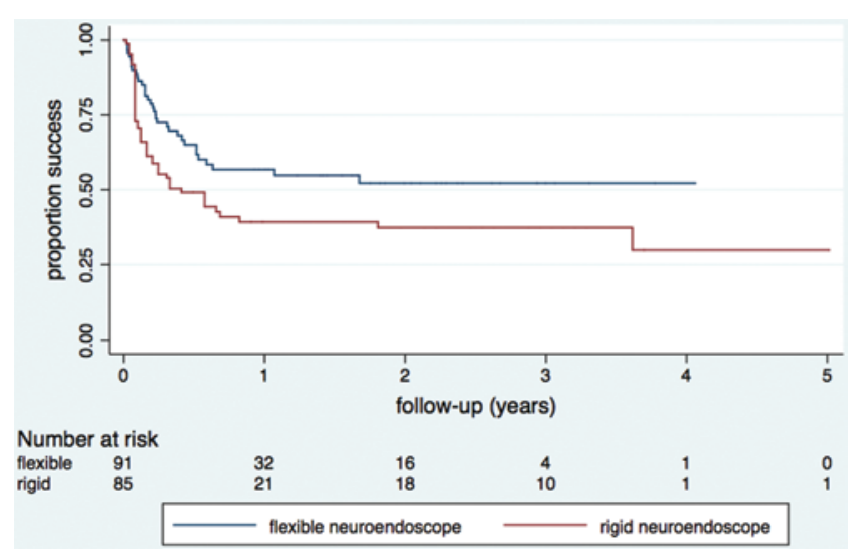

FIG. 2. Kaplan-Meier survival estimates for ETV/CPC success, by neuroendoscope type. Figure is available in color online only.

the median of 3.2 months was associated with treatment success (HR 0.58, 95\% CI 0.38-0.90, $p=0.014$; Fig. 6). Other variables, including patient sex and year of surgery, were not associated with significant differences in ETV/ CPC treatment outcome.

Crude comparison of the flexible and rigid neuroendoscopy cohorts revealed significant differences in patient characteristics and surgical case mix. Compared with the flexible neuroendoscopy cohort, the rigid neuroendoscopy cohort consisted of significantly more infants with prior diversion (38.8\% vs $18.9 \%$ ) and prepontine scarring ( $29.4 \%$ vs $16.5 \%$ ). That is to say, the case mix of infants who underwent rigid neuroendoscopy for ETV/CPC exhibited less favorable baseline characteristics that independently predicted treatment failure.

\section{Propensity Score Analysis}

The unadjusted odds ratio (OR) of ETV/CPC failure for rigid neuroendoscopy, compared with flexible neuroendoscopy, was 2.40 (95\% CI 1.31-4.40, $\mathrm{p}=0.034)$. On survival analysis, the Cox proportional hazards model revealed an HR of 1.61 (95\% CI 1.05-2.48, $\mathrm{p}=0.031)$. Therefore, us-

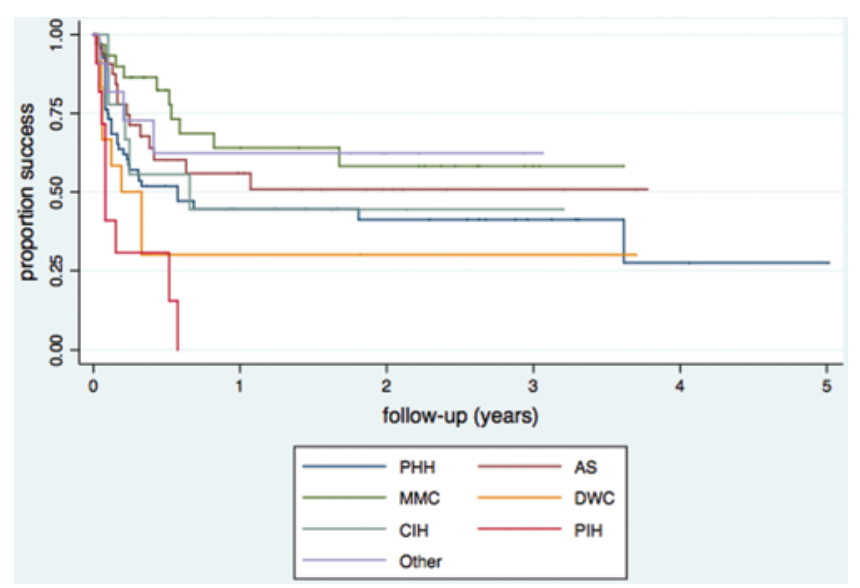

FIG. 3. Kaplan-Meier survival estimates for ETV/CPC success, by hydrocephalus etiology. $\mathrm{AS}=$ aqueductal stenosis; $\mathrm{ClH}=$ congenital idiopathic hydrocephalus; DWC = Dandy-Walker complex; MMC = myelomeningocele; $\mathrm{PHH}=$ posthemorrhagic hydrocephalus; $\mathrm{PIH}=$ postinfectious hydrocephalus. Figure is available in color online only. 


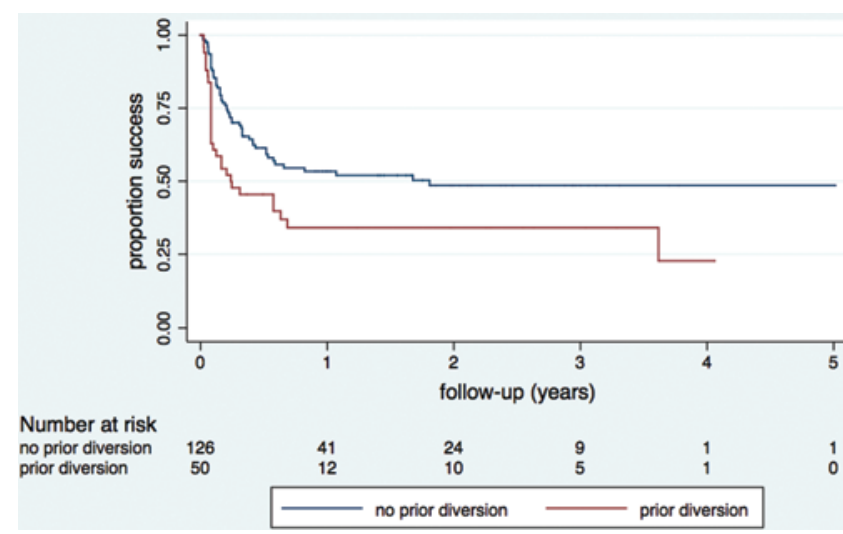

FIG. 4. Kaplan-Meier survival estimates for ETV/CPC success, by presence of prior CSF diversion. Figure is available in color online only.

ing both logistic regression and Cox proportional hazards regression, the unadjusted analyses reveal an association between rigid neuroendoscopy and poor outcomes.

However, because this was a nonrandomized group with likely confounders, propensity score matching was performed with neuroendoscopy type as the dependent variable. Five independent covariates were introduced into the propensity score model: age, sex, hydrocephalus etiology, prior CSF diversion, and presence of prepontine scarring. Age, etiology, and prior CSF diversion are the independent factors determining ETV success comprising the ETVSS. ${ }^{3}$ Scarring of the prepontine cistern has been independently found to determine success for ETV/CPC in both postinfectious and posthemorrhagic hydrocephalus. ${ }^{13,16}$

Propensity scores were generated, and distributions were assessed to be appropriate (Fig. 7). Model discrimination and calibration were calculated by the $\mathrm{C}$-statistic $(\mathrm{C}$ $=0.71$ ) and Hosmer-Lemeshow chi-square statistic (3.48; $\mathrm{p}$ $=0.90$ ), suggesting moderate discrimination and appropriate fit.

Decile-adjusted propensity scores using all 176 infants revealed that, compared with flexible neuroendoscopy, children undergoing rigid neuroendoscopy had a nonsignificant trend toward ETV/CPC failure, with an OR of $1.43(95 \%$ CI $0.72-2.86, \mathrm{p}=0.31)$. Propensity score 1 -to-1

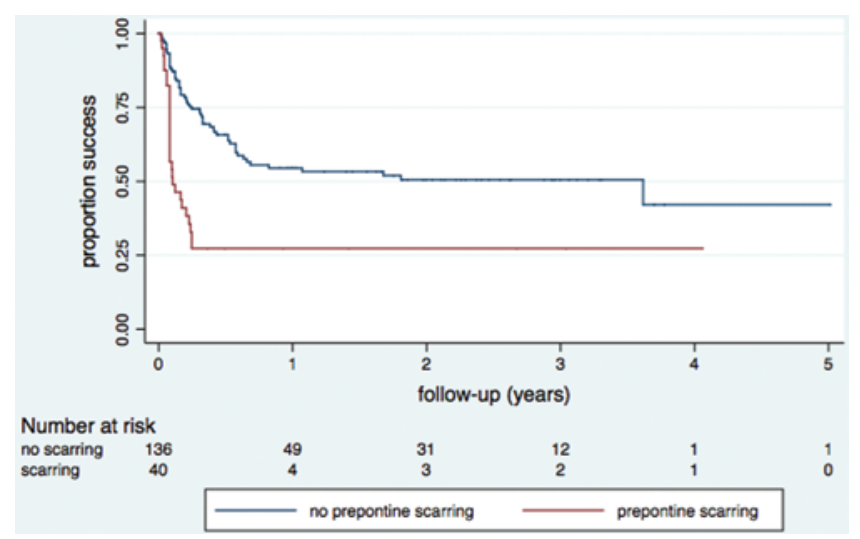

FIG. 5. Kaplan-Meier survival estimates for ETVICPC success, by the presence of prepontine scarring. Figure is available in color online only.

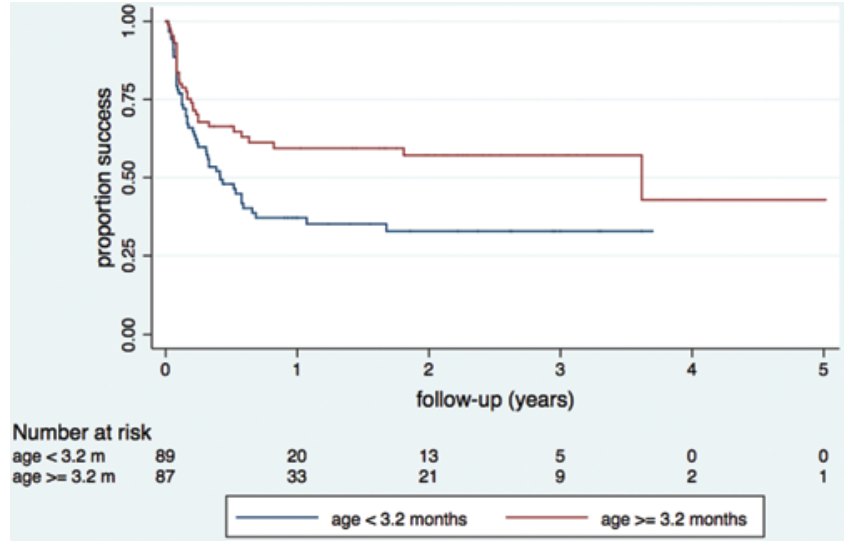

FIG. 6. Kaplan-Meier survival estimates for ETV/CPC success, by patient age. Figure is available in color online only.

nearest-neighbor matching was performed using calipers of 0.21 ( 0.25 times the standard deviation of the logit of the propensity scores) and involved 120 infants. A nonsignificant trend toward failure for rigid neuroendoscopy was present, with an OR of 1.31 (95\% CI 0.64-2.68, p = 0.47).

Our secondary analyses included propensity score stratification and covariate adjustment using the propensity score in Cox regression modeling. Controlling for propensity score deciles, the HR of ETV/CPC failure for rigid neuroendoscopy was 1.10 (95\% CI $0.69-1.73, \mathrm{p}=0.70$ ); the $p$ value was comparable to a log-rank test stratified on propensity score deciles $(\mathrm{p}=0.76)$.

\section{Discussion}

\section{Summary of Findings}

This analysis is the first paper comparing the effectiveness of flexible and rigid neuroendoscopy for ETV/CPC, using data from 2 high-volume neuroendoscopy centers in North America and contemporary cases from 2007 to 2014. Univariate Cox proportional hazards regression analysis of the pooled data demonstrated statistically significant determinants of ETV/CPC treatment success: older patient age, certain hydrocephalus etiologies (espe-

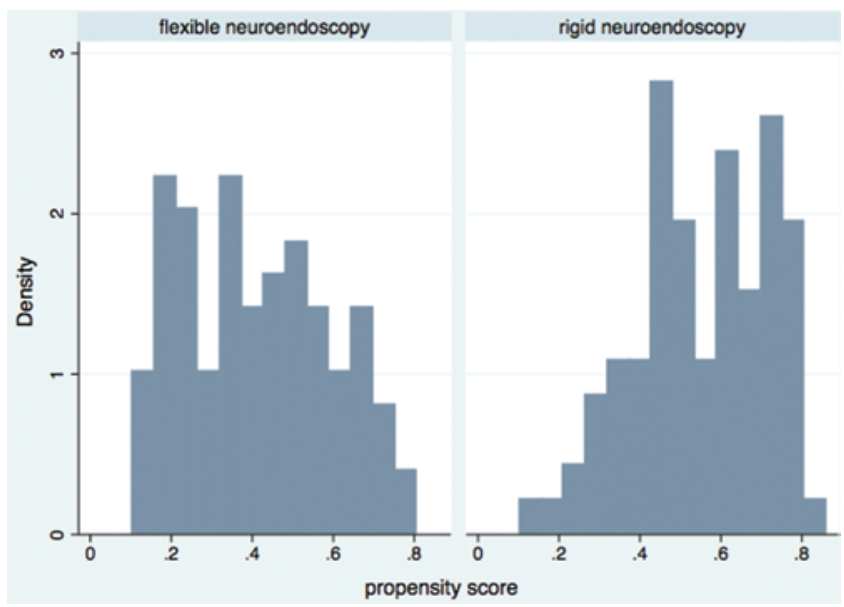

FIG. 7. Propensity score distribution by neuroendoscopy type. Figure is available in color online only. 
cially noninfectious etiologies), absence of prior diversion, and absence of prepontine scarring. Unadjusted analyses for logistic regression and Cox proportional hazards regression revealed worse outcomes associated with rigid neuroendoscopy. However, once adjusted for confounders using propensity scores, the apparent superiority of flexible neuroendoscopy was reduced to a nonsignificant trend for ETV/CPC outcome by endoscope type using both logistic regression and Cox regression analyses. Our analyses suggest that the different treatment outcomes reported between these flexible and rigid neuroendoscope cohorts are partially attributed to a difference in surgical case mix and other underlying risk factors, including different center-specific thresholds for attempting ETV/CPC in poorer surgical candidates and for abandoning ETV/CPC intraoperatively based on the presence of prepontine scarring. In the absence of a randomized controlled trial, this propensity score-adjusted multicenter, retrospective cohort study provides the best available evidence to inform the comparative effectiveness of flexible versus rigid neuroendoscopy techniques in ETV/CPC.

\section{Limitations of the Study}

There are a few important limitations of this study, both in the methodology of the study and in the generalizability of the results. First, data were collected from only 2 centers in North America, with all cases at 1 center completed by a single neurosurgeon, and treatment failure was determined by the treating surgeons in all cases, with no independent adjudication. Therefore, heterogeneity in surgeon- and center-specific surgical technique, center-specific patient characteristics, and the threshold for reoperation cannot be accounted for in this model. These are unmeasured confounders that may independently contribute to treatment outcome. Although propensity scores were used to balance risk factors associated with the case mix, unmeasured confounders were not accounted for. Second, prepontine scarring, a previously demonstrated factor in ETV/CPC success, was determined intraoperatively by the surgeon, and thus not standardized across the cohorts. The intrarater reliability of assessment of prepontine scarring has not been determined. Third, our results depend on the surgeon's ability to estimate the completeness of CPC and their expertise in performing extensive CPC. Differences in outcome may be attributed to completeness of CPC instead of specific neuroendoscope type, as suggested by other studies. ${ }^{6}{ }^{67}$ Lastly, the external generalizability in extrapolating our findings to other centers, especially those outside North America, is uncertain.

\section{Conclusions}

Although unadjusted analyses suggested worse ETV/ CPC success for infants treated by rigid neuroendoscopy, propensity score-adjusted analyses revealed that much of the difference is attributed to the case mix and other predictors of outcome. Larger sample observational studies or randomized controlled trials are required to provide evidence-based guidelines on ETV/CPC technique. Further analyses from other centers, especially if patients are randomized, might help elucidate the clinical effectiveness and cost-effectiveness of these 2 surgical techniques, and help set a gold standard for ETV/CPC.

\section{Acknowledgments}

This work was conducted with support from Harvard Catalyst, The Harvard Clinical and Translational Science Center (National Center for Research Resources and the National Center for Advancing Translational Sciences, NIH Award no. UL1 TR001102), and financial contributions from Harvard University and its affiliated academic health care centers. The content is solely the responsibility of the authors and does not necessarily represent the official views of Harvard Catalyst, Harvard University and its affiliated academic health care centers, or the NIH.

\section{References}

1. Bankole OB, Ojo OA, Nnadi MN, Kanu OO, Olatosi JO: Early outcome of combined endoscopic third ventriculostomy and choroid plexus cauterization in childhood hydrocephalus. J Neurosurg Pediatr 15:524-528, 2015

2. Beuriat PA, Szathmari A, Grassiot B, Plaisant F, Rousselle C, Mottolese C: Role of endoscopic third ventriculostomy in the management of myelomeningocele-related hydrocephalus: a retrospective study in a single French institution. World Neurosurg 87:484-493, 2016

3. Kulkarni AV, Drake JM, Mallucci CL, Sgouros S, Roth J, Constantini S: Endoscopic third ventriculostomy in the treatment of childhood hydrocephalus. J Pediatr 155:254-259, 259.e1, 2009

4. Kulkarni AV, Riva-Cambrin J, Browd SR, Drake JM, Holubkov R, Kestle JR, et al: Endoscopic third ventriculostomy and choroid plexus cauterization in infants with hydrocephalus: a retrospective Hydrocephalus Clinical Research Network study. J Neurosurg Pediatr 14:224-229, 2014

5. Ogiwara H, Uematsu K, Morota N: Obliteration of the choroid plexus after endoscopic coagulation. J Neurosurg Pediatr 14:230-233, 2014

6. Pindrik J, Rocque BG, Arynchyna AA, Johnston JM, Rozzelle CJ: Radiographic markers of clinical outcomes after endoscopic third ventriculostomy with choroid plexus cauterization: cerebrospinal fluid turbulence and choroid plexus visualization. J Neurosurg Pediatr 18:287-295, 2016

7. Scarff JE: Nonobstructive hydrocephalus; treatment by endoscopic cauterization of the choroid plexus; long term results. J Neurosurg 9:164-176, 1952

8. Stone SS, Warf BC: Combined endoscopic third ventriculostomy and choroid plexus cauterization as primary treatment for infant hydrocephalus: a prospective North American series. J Neurosurg Pediatr 14:439-446, 2014

9. Warf BC: Comparison of endoscopic third ventriculostomy alone and combined with choroid plexus cauterization in infants younger than 1 year of age: a prospective study in 550 African children. J Neurosurg 103 (6 Suppl):475-481, 2005

10. Warf BC: Congenital idiopathic hydrocephalus of infancy: the results of treatment by endoscopic third ventriculostomy with or without choroid plexus cauterization and suggestions for how it works. Childs Nerv Syst 29:935-940, 2013

11. Warf BC: Three steps forward and 2 steps back: the Echternach procession toward optimal hydrocephalus treatment. Neurosurgery 61 (Suppl 1): 105-110, 2014

12. Warf BC, Campbell JW: Combined endoscopic third ventriculostomy and choroid plexus cauterization as primary treatment of hydrocephalus for infants with myelomeningocele: long-term results of a prospective intent-to-treat study in 115 East African infants. J Neurosurg Pediatr 2:310-316, 2008

13. Warf BC, Campbell JW, Riddle E: Initial experience with combined endoscopic third ventriculostomy and choroid plexus cauterization for post-hemorrhagic hydrocephalus of 
prematurity: the importance of prepontine cistern status and the predictive value of FIESTA MRI imaging. Childs Nerv Syst 27:1063-1071, 2011

14. Warf BC, Dagi AR, Kaaya BN, Schiff SJ: Five-year survival and outcome of treatment for postinfectious hydrocephalus in Ugandan infants. J Neurosurg Pediatr 8:502-508, 2011

15. Warf BC, Dewan M, Mugamba J: Management of DandyWalker complex-associated infant hydrocephalus by combined endoscopic third ventriculostomy and choroid plexus cauterization. J Neurosurg Pediatr 8:377-383, 2011

16. Warf BC, Kulkarni AV: Intraoperative assessment of cerebral aqueduct patency and cisternal scarring: impact on success of endoscopic third ventriculostomy in 403 African children. J Neurosurg Pediatr 5:204-209, 2010

17. Warf BC, Mugamba J, Kulkarni AV: Endoscopic third ventriculostomy in the treatment of childhood hydrocephalus in Uganda: report of a scoring system that predicts success. J Neurosurg Pediatr 5:143-148, 2010

18. Warf BC, Stagno V, Mugamba J: Encephalocele in Uganda: ethnic distinctions in lesion location, endoscopic management of hydrocephalus, and survival in 110 consecutive children. J Neurosurg Pediatr 7:88-93, 2011

19. Warf BC, Tracy S, Mugamba J: Long-term outcome for endoscopic third ventriculostomy alone or in combination with choroid plexus cauterization for congenital aqueductal stenosis in African infants. J Neurosurg Pediatr 10:108-111, 2012

20. Weil AG, Fallah A, Chamiraju P, Ragheb J, Bhatia S: Endo- scopic third ventriculostomy and choroid plexus cauterization with a rigid neuroendoscope in infants with hydrocephalus. J Neurosurg Pediatr 17:163-173, 2016

\section{Disclosures}

The authors report no conflict of interest concerning the materials or methods used in this study or the findings specified in this paper.

\section{Author Contributions}

Conception and design: Kulkarni, Wang, Fallah. Acquisition of data: all authors. Analysis and interpretation of data: all authors. Drafting the article: all authors. Critically revising the article: Kulkarni, Wang, Stone, Weil, Warf, Ragheb, Bhatia. Reviewed submitted version of manuscript: Kulkarni, Wang, Stone, Weil, Warf, Ragheb, Bhatia. Approved the final version of the manuscript on behalf of all authors: Kulkarni. Statistical analysis: Kulkarni, Wang, Fallah, Warf, Ragheb, Bhatia. Administrative/ technical/material support: Kulkarni, Wang. Study supervision: all authors.

\section{Correspondence}

Abhaya V. Kulkarni, Department of Surgery, The Hospital for Sick Children, 555 University Ave., Toronto, ON M5G 1X8, Canada.email: abhaya.kulkarni@sickkids.ca. 